\title{
Application of an HPLC-FTIR Modified Thermospray Interface for Analysis of Dye Samples
}

\author{
Mohammad A MotTaleB ${ }^{\dagger}$ and David LiTtLejohn \\ Department of Pure and Applied Chemistry, University of Strathclyde, Thomas Graham Building, \\ 295 Cathedral Street, Glasgow G1 1XL, United Kingdom
}

\begin{abstract}
Previously, we developed a reversed-phase HPLC method compatible to high performance liquid chromatography diffuse reflectance Fourier-transform infrared (HPLC-FTIR) thermospray interface for the analysis of dyes. Dye separation achieved with a mixed-mode (SCX-ODS) column using a small gradient (90 to $80 \%$ water with acetic acid) and $\mathrm{pH} 3.25$; 10 to $20 \%$ acetonitrile was considered to be suitable for HPLC-FTIR. A constant-voltage setting for the thermospray temperature $\left(227^{\circ} \mathrm{C}\right)$ was successfully used for this gradient condition. The HPLC-separated components deposited as a series of concentrated spots on a moving tape were scanned by specially developed HPLC-FTIR software. Excellent repeatability of the thermospray deposition FTIR chromatograms and IR spectra was obtained. The interface-derived spectra of the separated components of formulated and purified reactive dyes were compared and differences in spectral features were observed.
\end{abstract}

(Received August 21, 2000; Accepted November 29, 2000)

Commercially, various kinds of dyes are available. Acidic dye is one of them, and is extensively used to color a number of materials, including foods, drinks, cosmetics, water-based paints, inks, leather and a wide range of fibers, e.g., wool, cotton, viscose, nylon, silk and modified acrylics ${ }^{1}$ etc. Thinlayer chromatography (TLC) is a widely used technique for examining the dye extracts and ink. ${ }^{2}$ In some instances, an improved resolution at the desired sensitivity was reported by using high-performance TLC. ${ }^{3}$ Although these approaches improve the sample discrimination, the chemical and structural differences between dyes are not fully exploited. ${ }^{4}$ An alternative approach is the combination of TLC with surfaceenhanced resonance Raman scattering used for dye analysis, which provides better discrimination and semi-quantitative analysis; fluorescence problems are also experienced. ${ }^{5}$ Diffuse reflectance Fourier transform infrared (DRIFT) and photoacoustic absorption spectroscopy (PAS) are widely used for dye characterisation. Griffiths et al. ${ }^{6}$ compared the DRIFT and PAS results and concluded that DRIFT is the more rapid method to obtain the IR spectrum from scattering surfaces. Specific applications of DRIFT have been investigated to analyze ball-point pen inks, ${ }^{7}$ cellulose fibers ${ }^{8}$ etc. It has also been reported that the DRIFT could effectively discriminate differently colored commercial cotton fabrics. ${ }^{9}$ However, there are some problems when measuring DRIFT spectra from a TLC plate. For example, components may undergo chemical changes during the separation. Consequently, the spectral results arising from these isolates can tend to be misleading.

High-performance liquid chromatography (HPLC) has become a popular method for the analysis of dyes. This

† To whom correspondence should be addressed.

M. A. M. present address: Department of Pharmacy, Dongduk Women's University, 23-1 Sungbuk-ku, Seoul 136-714, South Korea. technique has been used to analyze dyes extracted from polyester fibers, ${ }^{10}$ acidic dyes from wool fibers, ${ }^{11}$ basic dyes from acrylic fibers, ${ }^{12}$ natural and early synthetic dyes from archaeological textiles ${ }^{13}$ with a photodiode array and multiwavelength detection. Comparisons between HPLC and TLC have concluded that HPLC provides better sensitivity and resolution. ${ }^{10-12}$ In order to identify dye components separated by HPLC, various procedures have been used, including absorbance ratioing, ${ }^{14}$ peak purity parameters $^{15}$ and complementary chromaticity co-ordinate ${ }^{16}$ value. These approaches are able to provide limited characteristic properties of dyes. It is well established that the hyphenated techniques are the most powerful analytical tool for the separation and characterization of complex mixtures. Capillary HPLC-mass spectrometry (MS) and micellar electrokinetic chromatography (MEKC) have been reported for the analysis of synthetic dyes, ${ }^{17}$ and it was found that MEKC provides excellent separation and quantitation of dyes, while capillary HPLC-MS provides a confirmatory tool. Because MS is very expensive, many laboratories do not possess HPLC-MS facilities.

A preliminary study of reactive dyes (Fig. 1) was performed by HPLC-MS; ${ }^{18}$ the purified dye showed a different chromatographic pattern to that of the formulated one, which was surprising, because some common peaks were expected, especially for the parent dye component (Fig. 1(a)). It was thought that the purified dye might be hydrolyzed (Figs. 1(b) and (c)). We recently reported the development of HPLC to FTIR coupling using a modified thermospray interface. ${ }^{19}$ This coupled technique was employed to analyze the linear alkylbenzene sulfonate, ${ }^{20,21}$ river-water residues, ${ }^{22}$ polystyrene samples $^{23}$ and pesticides. ${ }^{24}$ Because HPLC-FTIR can provide functional-group features, ${ }^{19,20}$ it may also provide complementary information to HPLC-MS for dyes. It was of interest to access whether the provided functional-group features by FTIR could give sufficient information to explain 
(a)<smiles>CCOc1nc(Cl)nc(Nc2c(C)c([Se])c(C)c(NC3C=C4C(=O)c5ccccc5C(=O)C4=C(N)C3[Se])c2C)n1</smiles>

(b)

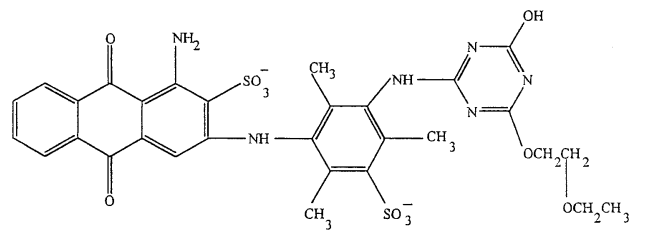

(c)

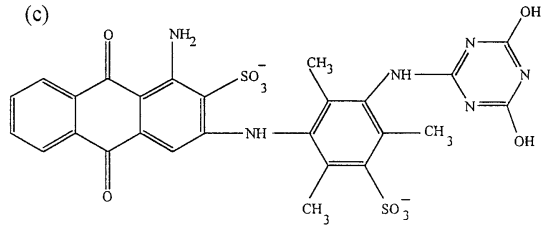

Fig. 1 The structure of the formulated reactive dyes: (a) parent reactive blue 74 , (b) singly hydrolyzed reactive blue 74 and (c) doubly hydrolyzed reactive blue 74 .

the reasons for the different chromatograms given by the formulated and purified reactive dyes. In the HPLC-FTIR thermospray interface, some problems were also experienced concerning the use-phase modifiers in the HPLC eluent. ${ }^{19-25}$ In order to analyze dye samples by HPLC-FTIR thermospray, a small-gradient HPLC method has been developed. ${ }^{25}$ This paper describes the applicability of an HPLC-FTIR modified thermospray interface for dye analysis. A comparison of UV and FTIR detection in HPLC analysis has also been described.

\section{Experimental}

\section{Chromatography and spectroscopy}

A Philips PU 4100 liquid chromatograph (Cambridge, UK), equipped with a Rheodyne (Model 7125) syringe loading injector (Berkeley, CA, USA) and a $20 \mu \mathrm{L}$ sample loop was used. The hypersil SCX DUET $(150 \times 4.6 \mathrm{~mm}$ i.d. $)$ Shandon 5 mixed (1:1) $\mathrm{C}_{18}$ :sulfonic acid was used analytical column. A Philips PU 4110 UV-VIS detector was used to monitor the dye samples at $260 \mathrm{~nm}$ prior to FTIR detection. Distilled water and acetonitrile were used as the mobile phase and water was degassed continuously by helium. Acetic acid was used as phase additives in the aqueous phase and passed through the

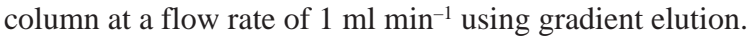

The IR spectrometric data were obtained by using a Philips PU 9800 series FTIR spectrometer equipped with a SpectraTech diffuse reflectance accessory (Stamford, CT, USA) and a deturiated triglycine sulfate (DTGS) detector. The IR data were collected at 30 scans per min using a resolution of $16 \mathrm{~cm}^{-1}$. These data were stored using standard Philips FTIR computer software. After each analysis, interferograms were transformed to IR transmittance spectra or Kubelka Munk spectrum ${ }^{26}$ by using a fast Fourier transform algorithm.

\section{An HPLC-FTIR interface}

A simplified diagram of the HPLC-FTIR interface is shown in Fig. 2. Column effluents were passed through a thermospray assembly where the eluent was desolvated by evaporation and

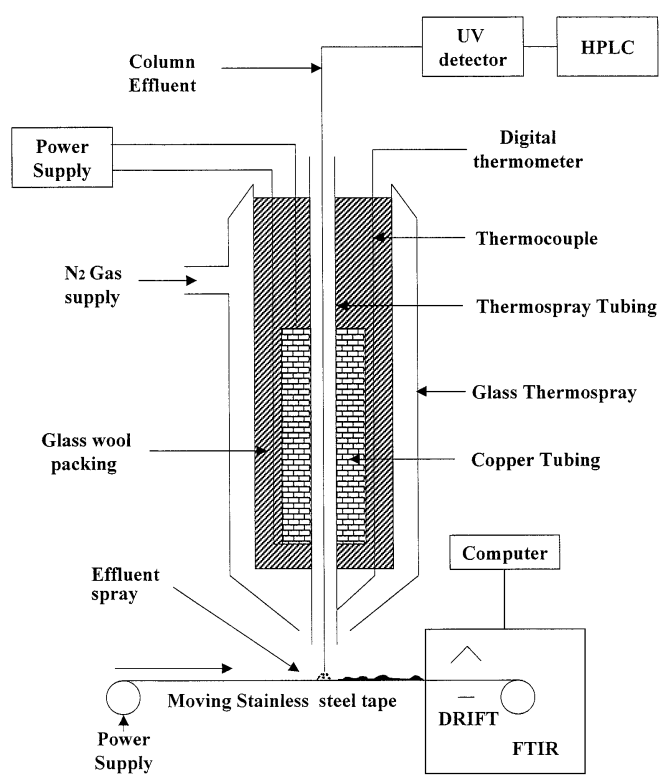

Fig. 2 A simplified schematic diagram of HPLC-FTIR thermospray interface assembly.

the solutes were deposited as a series of spots on a moving surface of a stainless-steel belt or tape. IR spectra of the deposited solutes were collected by scanning and by focusing an IR beam on a chromatographic spot on a moving surface of a stainless-steel tape. Background spectra were obtained from a clean part of the tape prior to the area of interest. Specially developed Philips HPLC-FTIR chromatogram software was used to process these data and to construct FTIR chromatograms. These were obtained by calculating the integrated IR absorbance across a wavenumber interval as a function of time. The wavenumber intervals 3550 to $3200 \mathrm{~cm}^{-1}$ and 1700 to $1500 \mathrm{~cm}^{-1}$ were used to construct the IR chromatograms, representing the $\mathrm{N}-\mathrm{H}$ and $\mathrm{C}=\mathrm{O}$ vibration, respectively. Standard spectra were prepared using $\mathrm{KBr}$ discs. These were produced by thoroughly grinding a small amount of solute (about 1\% sample) in $\mathrm{KBr}$. The standard spectra were obtained by ratioing these spectra to that of a $\mathrm{KBr}$ disc containing no sample. The spectrum of the $\mathrm{KBr}$ was stored as a background file. Details concerning on the development and description of the HPLC-FTIR interface have been reported. ${ }^{19-21}$ The dye samples were obtained from Zeneca, Brixham Environmental Laboratory, U.K. Dye solution was prepared by dissolving an appropriate amount of dye with 1:1 methanol: acetonitrile in a $10 \mathrm{ml}$ volumetric flask to give a working concentration of $800 \mathrm{mg} \mathrm{l}^{-1}$. All chemicals, solvent and reagents were obtained from Merck and Fisons Scientific Ltd. with HPLC or analytical grade.

\section{Results and Discussion}

\section{Gradient elution and thermospray deposition}

The separation achieved with a mixed-mode SCX-ODS column using a small gradient of $90-80 \%$ acidified water (10$20 \%$ acetonitrile) with a run time of $10 \mathrm{~min}$ and a hold time of 8 min at the final percentage were considered to be a satisfactory condition for the analysis of dyes by an HPLC-FTIR thermospray interface. ${ }^{25}$ Fortunately, only a slight change in the thermospray temperature (and deposition conditions) was 


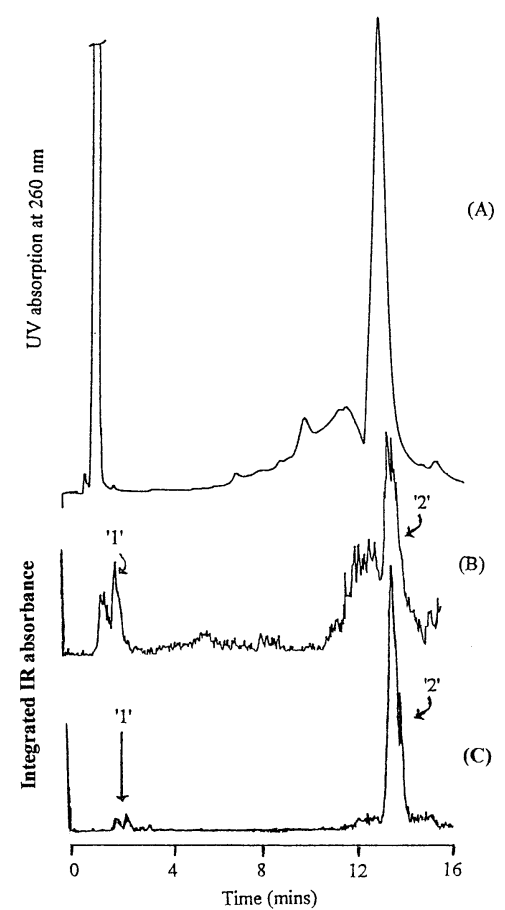

Fig. 3 HPLC chromatograms for (A) UV detection at $260 \mathrm{~nm}$, (B) FTIR detection $\left(v\right.$ window $\left.=3500-3200 \mathrm{~cm}^{-1}\right)$ and $(\mathrm{C})$ FTIR detection $\left(v\right.$ window $\left.=1700-1500 \mathrm{~cm}^{-1}\right)$ of formulated dye. Conditions: [(i) chromatography] solvents, (A) water (pH 3.25 with acetic acid) and (B) acetonitrile, gradient elution of $90-80 \%$ (A)

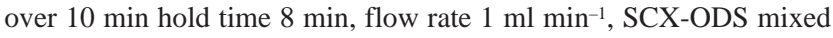
mode column, $150 \times 4.6 \mathrm{~mm}$ i.d., $20 \mu \mathrm{l}$ loop, $\mathrm{UV}$ detection at 260 $\mathrm{nm}$, dye conc. $800 \mathrm{mg} \mathrm{l}^{-1}$; [(ii) interface] auxiliary heating tape voltage $190 \mathrm{~V}$, temperature $110^{\circ} \mathrm{C}$, thermospray voltage $37.7 \mathrm{~V}$, total temperature recorded $227^{\circ} \mathrm{C}$, thermospray height $0.3 \mathrm{~cm}$, substrate speed $0.31 \mathrm{~cm} \mathrm{~min}{ }^{-1}$ for deposition and $0.1 \mathrm{~cm} \mathrm{~min}^{-1}$ for data acquisition and nitrogen gas flow rate $6 \mathrm{~L} \mathrm{~min}^{-1}$.

observed when $90 \%$ acidified water-10\% acetonitrile was used. With this gradient, the required thermospray temperature for satisfactory desolvation was $227^{\circ} \mathrm{C}$. At the end of the gradient change $(10 \mathrm{~min})$, a slight increase in the thermospray temperature $\left(0.5^{\circ} \mathrm{C}\right)$ was observed, which did not affect the solute deposition. However, a greater change in the thermospray temperature was obtained within the hold period $(8$ min). After the initial $3 \mathrm{~min}$ of the hold period, it was seen that the thermospray temperature had increased up to $228.5^{\circ} \mathrm{C}$ and the eluent spray from the thermospray tip became narrower and almost dry. After the next $2 \mathrm{~min}$ of the hold period, the thermospray temperature increased to $230^{\circ} \mathrm{C}$ and no spray of solvent from the thermospray tip was observed and the thermospray temperature fluctuated between 230 and $238^{\circ} \mathrm{C}$. Sometimes, the back pressure of the HPLC pump also slightly increased during the fluctuation of temperature when about 10 depositions were carried out continuously, which may have been indicative that a small amount of material being deposited in the inner wall of the thermospray capillary tubing. Thus, although it was concluded that the single voltage temperature setting of the thermospray interface was just about satisfactorily for the gradient used, care was required to ensure proper operation.

\section{UV and FTIR chromatograms of the dye}

Figure 3 compares the HPLC chromatograms for (A) UV

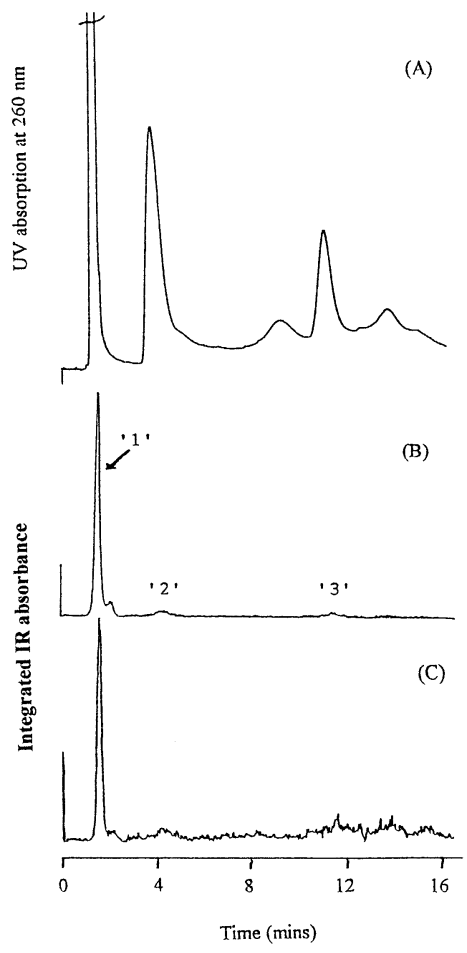

Fig. 4 HPLC chromatograms for (A) UV detection at $260 \mathrm{~nm}$, (B) FTIR detection $\left(v\right.$ window $=3500-3200 \mathrm{~cm}^{-1}$ and (C) FTIR detection $\left(v\right.$ window $\left.=1700-1500 \mathrm{~cm}^{-1}\right)$ of purified dye. Conditions are same as in Fig. 3 .

detection $(\lambda=260 \mathrm{~nm})$, (B) FTIR detection $(v$ window $=3550$ $\left.3200 \mathrm{~cm}^{-1}\right)$ and $(\mathrm{C})$ FTIR detection $(v$ window $=1700-1500$ $\mathrm{cm}^{-1}$ ) of formulated reactive dye. Although this shows similar features, some splitting of the peak with a retention time of 1.55 min is apparent in the FTIR chromatograms. This may be due to spreading of solute on the stainless-steel belt during thermospray deposition. ${ }^{19}$ The peak height of the first peak (elution time $1.55 \mathrm{~min}$ ) detected at $1700-1500 \mathrm{~cm}^{-1}$ is significantly smaller than that of the peak height detected at $3550-3200 \mathrm{~cm}^{-1}$. This is due to the difference in the magnitude of the respective IR absorbance band in the spectrum of the component eluted for $1.55 \mathrm{~min}$. The peak eluted for $14 \mathrm{~min}$ shows quite a strong IR absorbance signal in both FTIR chromatograms. Figure 4 shows the chromatograms obtained for purified dye with (A) UV detection at $260 \mathrm{~nm}$, (B) FTIR detection at $3550-3200 \mathrm{~cm}^{-1}$ and (C) FTIR detection at $1700-$ $1500 \mathrm{~cm}^{-1}$. This exhibits that there are five peaks in the UV chromatograms, whereas only three peaks can be seen in the FTIR plots. Although the first component eluted at $1.55 \mathrm{~min}$ exhibits strong absorption, the components at 4.25 and $11.3 \mathrm{~min}$ are only just detectable at the scale expansion used. This is also due to the difference in the magnitude of the IR band in the spectrum of the component eluted for $1.55 \mathrm{~min}$. The other two components eluted for 9.5 and 14 min were not detected in the FTIR detection. The solutes are probably present in small quantities, less than the detection limit of FTIR. A comparison of the UV chromatograms for formulated (Fig. 3A) and purified (Fig. 4A) dyes shows a common feature at a retention time of $1.55 \mathrm{~min}$. The IR spectra of the two components are given later. The peak obtained for purified dye at $4.25 \mathrm{~min}$ in Fig. 4A, is not featured in Fig. 3A for formulated dye. Also, the formulated dye shows a large UV absorption peak at $13.8 \mathrm{~min}$ (Fig. 3A), whereas there is a small peak at this retention time in the 


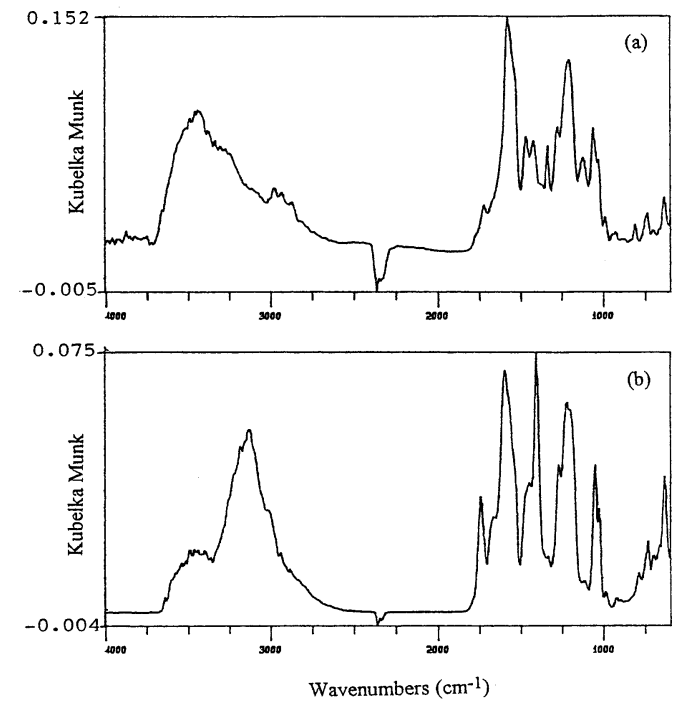

Fig. 5 Standard FTIR spectra for (a) the unseparated formulated dye and (b) the unseparated purified dye.

chromatogram of the purified dye (Fig. 4A). Other differences exist in the chromatograms, between 10 and 16 min, suggesting that different compounds may be present in the formulated and purified dyes. This possibility was considered further by a more detailed comparison of the IR spectra of the peaks. Blank chromatograms for UV and IR detection were obtained with each set of sample analyzed; no peaks were detected (not shown).

\section{FTIR analysis of dyes}

As stated before, the formulated and purified dye may contain different types of impurities. Figure 5 compares the IR spectra of unseparated formulated and purified reactive dyes. In these spectra, the main absorption peaks are obtained at 3600-2800 $\mathrm{cm}^{-1}$ and $1750-1000 \mathrm{~cm}^{-1}$. The peak at $1700-1550 \mathrm{~cm}^{-1}$ in (a) and (b) spectra can be assigned to the $\mathrm{C}=\mathrm{O}$ stretch. The other peaks in spectrum (b) are of relatively higher magnitude than spectrum (a). However, in both dye spectra, the band at 1300 $1150 \mathrm{~cm}^{-1}$ can be assigned as the $\mathrm{SO}_{3}$ group, as confirmed by a small absorption band at $1050-1000 \mathrm{~cm}^{-1}$. The absorption band of $2950-2800 \mathrm{~cm}^{-1}$ in formulated dye spectrum (a) was assigned to the $\mathrm{C}-\mathrm{H}$ stretching vibration. However, this band was obscured in the spectrum of purified dye (b), probably by bands of hydrogen-bonded $\mathrm{N}-\mathrm{H}$ stretching, that corresponds to $3550-3150 \mathrm{~cm}^{-1}$. The bands between 1480 and $1300 \mathrm{~cm}^{-1}$ can be assigned for $-\mathrm{CH}_{2},-\mathrm{CH}_{3}$ deformation vibrations. A comparison of the spectra clearly shows that the formulated dye is impure, because the peaks in the spectrum of the formulated dye lie between the major absorbance maxima in the spectrum of the purified dye. Figure 6 compares the IR spectra of the major components of the formulated dye, as isolated by HPLC. Spectra (a) and (b) were obtained from peaks ' 1 ' and ' 2 ' in Fig. 3(B) corresponding to retention times of 1.55 and $14 \mathrm{~min}$ in FTIR chromatograms. These spectra showed different patterns. The relative sizes of the peaks in spectra (a) and (b) are quite different to each other in the ranges of $3600-2800 \mathrm{~cm}^{-1}$ and $1750-1000 \mathrm{~cm}^{-1}$. However, it can be concluded that the component giving spectrum (b) is the main constituent of the formulated dye, and is different from the component which was eluted at $1.55 \mathrm{~min}$, giving spectrum (a). Spectra (c) and (d) were obtained for the components, giving peaks ' 1 ' and ' 2 ' at

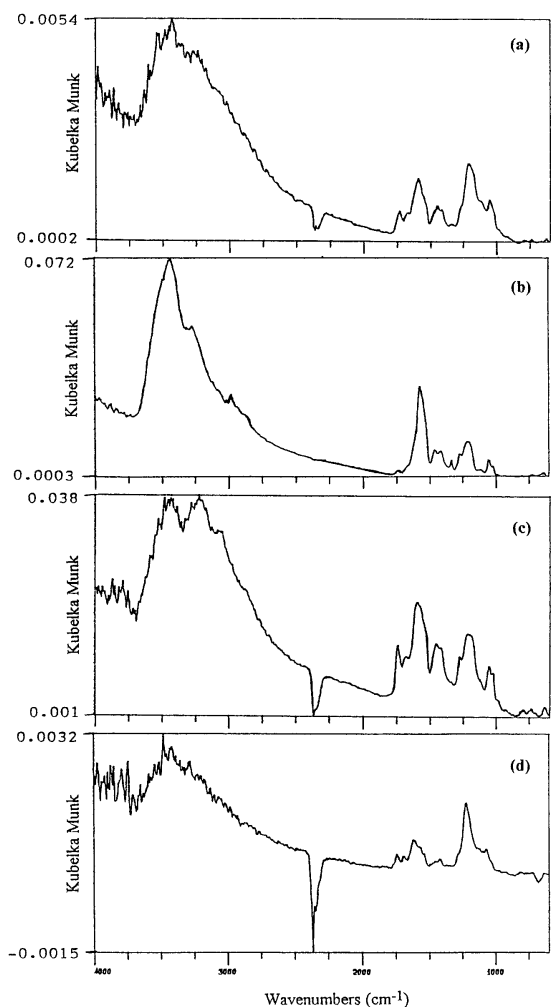

Fig. 6 HPLC-FTIR interface derived IR spectra; spectra (a), (b) for separated components of the formulated dye, taken from the peaks ' 1 ' and ' 2 ' in Fig. 3(B), respectively and spectra (c), (d) for separated components of the purified dye, taken from the peaks ' 1 ' and ' 2 ' in Fig. 4(B), respectively. Conditions are same as in Fig. 3.

retention times of 1.55 and 4.35 min, respectively, in the FTIR chromatograms (Fig. 4(B)). The component with a retention time of $1.55 \mathrm{~min}$, giving spectrum (c), is clearly the most important part of the dye, but by no means the only one present. The second component with an elution time of $4.35 \mathrm{~min}$, giving spectrum (d), would appear to contribute little to the spectrum of the unseparated purified dye (Fig. 5(b)). The IR spectrum of the component with a retention time of $11.8 \mathrm{~min}$ (not shown) had almost identical spectral features to those of spectrum (d). Interestingly, neither spectrum (c) nor (d) accounts for the peaks at $750 \mathrm{~cm}^{-1}$ that exist in the spectrum (Fig. 5(b)). The comparison of spectra in Figs. 6(a) and (c) clearly shows that the first components in the formulated and purified dyes are two different compounds, although they were eluted at the same retention time $(1.55 \mathrm{~min})$ in the UV chromatograms. This is one of the major advantages of FTIR detection for the identification or characterisation of unknown compounds in HPLC analysis.

\section{Repeatability of thermospray deposition, IR chromatograms and spectra}

Figure 7 exhibits the repeatability of UV and FTIR detection chromatograms of formulated dye. This was done by carrying out duplicate depositions of HPLC isolated components under the same experimental conditions. These show excellent repeatability, similar chromatographic features and retention times. In addition, it can also be seen that the splitting of peak (peak ' 1 ') seems to be reproducible, but relative strength of peak ' 2 ' in Fig. 7(C) is higher than that of Fig. 7(B). This may be a reason for solute spreading during thermospray deposition. 


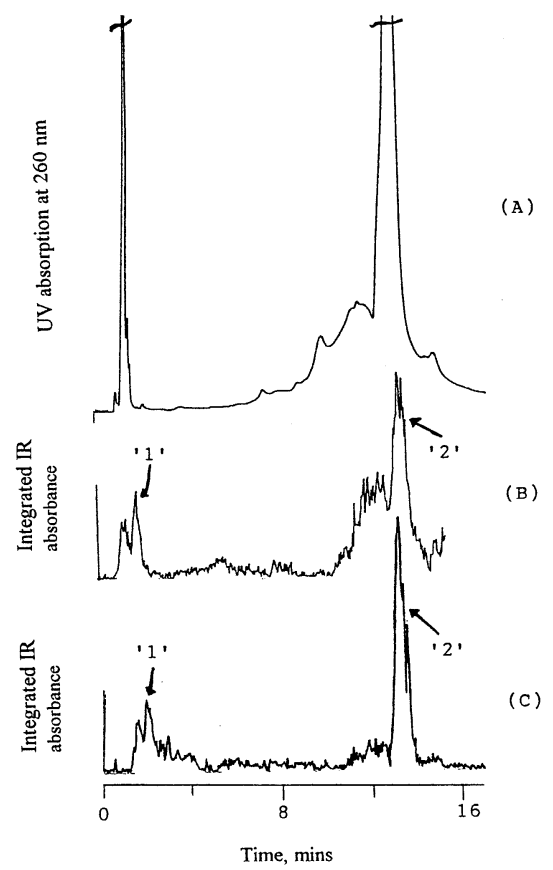

Fig. 7 HPLC chromatograms for (A) UV detection at $260 \mathrm{~nm}$, and (B) and (C) FTIR detection $\left(v\right.$ window $\left.=3500-3200 \mathrm{~cm}^{-1}\right)$ showing the repeatability of the first and duplicate depositions of the formulated dye. Conditions are same as in Fig. 3.

The IR chromatograms were constructed between at 3550 and $3200 \mathrm{~cm}^{-1}$. IR spectra (a), (b) and (c), (d) in Fig. 8 correspond to peaks ' 1 ' and '2', respectively, in the FTIR plots in Figs. 7(B) and $(\mathrm{C})$ for the first and duplicate depositions of the formulated dye. In general, there is a good agreement between the spectral features in duplicate spectra (a), (b) and (c), (d) in Fig. 8. The repeatability of the UV and FTIR detection chromatograms and the IR spectra of purified dye for duplicate depositions were also investigated. Similar chromatographic behaviors and spectral features were obtained. These are not shown. Hence, it was concluded that the repeatability of thermospray deposition for dye samples and their analysis by HPLC-FTIR were satisfactory.

In conclusion, thermospray deposition using gradient elution with a small change in the eluent composition (e.g., 90 to $80 \%$ acidified water, $\mathrm{pH} 3.25,10$ to $20 \%$ acetonitrile, for $10 \mathrm{~min}$ for 8 min hold) could be used to separate of dyes at one thermospray temperature setting. A slight variation of the thermospray temperature $\left(0.5^{\circ} \mathrm{C}\right)$ was obtained within the selected gradient, which was not harmful for the deposition of solutes. This is the first report concerning the use of gradient elution for thermospray deposition of dye samples and analysis by HPLC-FTIR with diffuse reflectance detection. The components detected by UV and FTIR, eluted at $1.55 \mathrm{~min}$ in the UV chromatogram, but gave different spectra obtained from FTIR chromatograms. It was thus concluded that there could be two different compounds in the formulated and purified dyes that gave the same retention time. Standard spectra of formulated and purified dyes showed a difference in the spectral features and also exhibited a difference in their features for deposited solute spectrum. The repeatability of the thermospray depositions, UV and FTIR chromatograms and IR spectra was investigated and found to be good. The HPLC-FTIR showed poor sensitivity for dye analysis. However, the analyzed dye

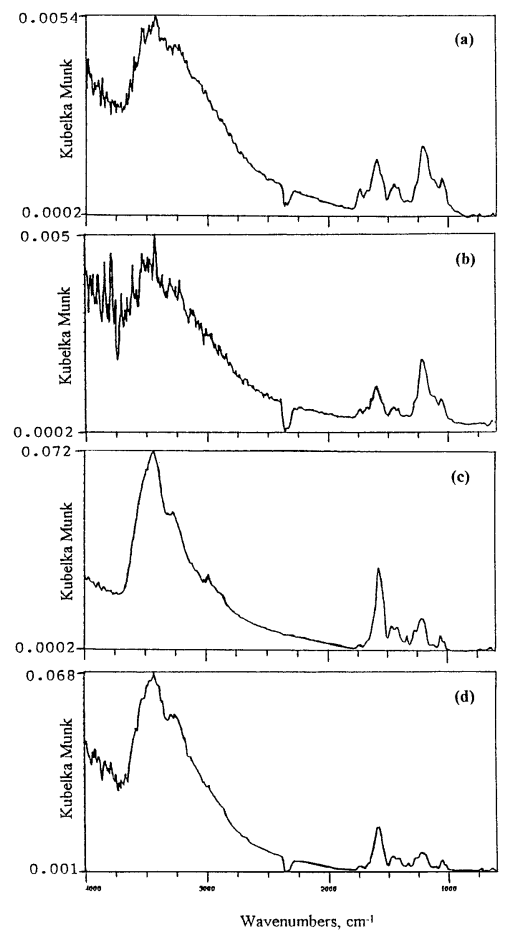

Fig. 8 HPLC-FTIR interface derived IR spectra; spectra (a), (b) for separated component ' 1 ' in FTIR chromatograms (Figs. 7(B) and (C)), the first and duplicate depositions, respectively and spectra (c), (d) for separated component '2' in FTIR chromatograms (Figs. 7(B) and $(\mathrm{C})$ ), the first and duplicate depositions, respectively of the formulated dye. Conditions are same as in Fig. 3.

molecules may be partially/fully hydrolyzed by photodegradation in solution. A further HPLC-MS investigation is going on this issue.

\section{Acknowledgements}

The author would like to thank Dr. M. Hetheridge, Zeneca, Environmental Laboratory, Brixham, UK for supplying the dye samples and other supplementary information.

\section{References}

1. D. K. Laing, R. J. Dudley, R. Rickard, and D. C. Bennett, Forensic Sci. Int., 1991, 50, 23.

2. O. P. Jasuja, A. K. Singla, and B. L Seema, Forensic Sci. Int., 1989, 42, 255.

3. K. M. Varshney, T. Jettapa, and T. R. Baggi, Forensic Sci. Int., 1995, 72, 107.

4. G. Flodberg and J. Roeraade, J. Planar Chromatogr., 1995 , $8,10$.

5. C. H. Munro, W. E. Smith, and P. C. White, Analyst, 1995, 120,993

6. P. R. Griffiths and J. A. de Haseth, "Fourier Transform Infrared Spectrometry", 1986, Wiley Interscience, New York, 332.

7. R. A. Merrill and E. G. Bartick, J. Forensic Sci., 1992, 37, 528.

8. Y. Liu, S. Kokot, and T. J. Sambi, Analyst, 1998, 123, 633.

9. S. Kokot, N. A. Tuan, and L. Rintoul, Appl. Spectrosc., 
1997, $51,387$.

10. B. B. Wheals, P. C. White, and M. D. Paterson, J. Chromatogr., 1985, 350, 295.

11. D. K. Laing, R. Gill, and H. M. Bickley, J. Chromatogr., 1988, 442, 187.

12. R. M. E. Griffin, S. J. Speers, and T. G. Kee, J. Chromatogr. A, 1994, 674, 271.

13. C. H. Fischer, M. Bischof, and J. G. Rabe, J. Liq. Chromatogr., 1990, 13, 319.

14. P. C. White and B. B. Wheals, J. Chromatogr., 1984, 303, 211.

15. P. C. White and T. Catterick, Analyst, 1990, 115, 919.

16. P. C. White, Chromatogr. and Anal., 1994, June/July, 9.

17. W. C. Brumley, C. M. Brownrigg, and A. H. Crange, J. Chromatogr. A, 1994, 680, 635.
18. M. Hetheridige, personal communication, unpublished data.

19. M. A. Mottaleb, Anal. Sci., 1999, 15, 57.

20. M. A. Mottaleb, Mikrochim. Acta, 1999, 132, 31.

21. M. A. Mottaleb, Anal. Sci., 1999, 15, 1137.

22. M. A. Mottaleb, Orient. J. Chem., 1999, 15, 35.

23. M. A. Mottaleb, B. G. Cooksey, and D. Littlejohn, Fresenius J. Anal. Chem., 1997, 358, 536.

24. J. C. Jones, D. Littlejohn, and P. R. Griffiths, Appl. Spectrosc., 1999, 53, 792.

25. M. A. Mottaleb and D. Littlejohn, Anal. Sci., submitted.

26. R. W. Frei and J. D. MacNeil, "Diffuse Reflectance Spectroscopy in Environmental Problem-Solving", 1973, CRC press. 